\title{
Two-photon final states in peripheral heavy ion collisions
}

\author{
A. A. Natale, C. G. Roldão, and J. P. V. Carneiro \\ Instituto de Física Teórica, Universidade Estadual Paulista, Rua Pamplona 145, 01405-900 São Paulo, SP, Brazil
}

(Received 13 July 2001; published 30 November 2001)

\begin{abstract}
We discuss processes leading to two photon final states in peripheral heavy ion collisions at RHIC. Due to the large photon luminosity we show that the continuum subprocess $\gamma \gamma \rightarrow \gamma \gamma$ can be observed with a large number of events. We study this reaction when it is intermediated by a resonance made of quarks or gluons and discuss its interplay with the continuum process, verifying that in several cases the resonant process overwhelms the continuum one. It also investigated the possibility of observing a scalar resonance (the $\sigma$ meson) in this process. Assuming for the $\sigma$ the mass and total decay width values recently reported by the E791 Collaboration we show that RHIC may detect this particle in its two photon decay mode if its partial photonic decay width is of the order of the ones discussed in the literature.
\end{abstract}

DOI: 10.1103/PhysRevC.65.014902

PACS number(s): 25.75.-q, 13.60.-r, 14.40.Cs

\section{INTRODUCTION}

In the Relativistic Heavy-Ion Collider (RHIC) operating at the Brookhaven Laboratory beams of heavy ions are colliding with the main interest in the search of a quark-gluon plasma in central nuclear reactions. In addition to this important feature of heavy-ion colliders, peripheral collisions may give rise to a huge luminosity of photons opening the possibilities of studying two-photon and other interactions as discussed by several authors [1].

In order to study peripheral $\gamma-\gamma$ heavy-ion collisions we have to pay attention to two important facts, which are fundamental to determine the kind of physics that is truly accessible in these interactions. The first one is to avoid events where hadronic particle production overshadows the $\gamma-\gamma$ interaction, i.e., events where the nuclei physically collide (with impact parameter $b<2 R_{A}, R_{A}$ being the nuclear radius) are excluded from calculations of the usable luminosity. This exclusion has been discussed by Baur [2] and can be handled as described by Cahn and Jackson [3]. The second point to remember is that the photons will carry only a small fraction of the ion momentum, favoring low mass final states. Therefore hadronic resonances with masses up to a few $\mathrm{GeV}$ can be produced at large rates [4,5], as well as any two-photon process not leading to extremely heavy final states.

One of the purposes of our work was motivated by the possibility of measurement of the continuum process $\gamma \gamma$ $\rightarrow \gamma \gamma$, see Fig. 1, as suggested in Ref. [4], as well as the resonant one $\gamma \gamma \rightarrow R \rightarrow \gamma \gamma$ which was predicted in Ref. [5] as a clear signal for resonances made of quarks or gluons. The continuum $\gamma \gamma \rightarrow \gamma \gamma$ reaction is interesting per se but is also a background for the resonant process. This last one is quite important to be observed because it involves only the electromagnetic coupling of the resonance. Its knowledge with high precision is very useful, for instance, to unravel the possible amount of mixing in some glueball candidates [6], complementing the information obtained through the observation of hadronic decays. Another interesting study is the possible production of a light scalar meson $(\sigma)$.

The E-791 experiment recently reported that the $\sigma$ meson has been observed in its $\pi \pi$ decay mode [7], which is the same final state questioned over many years as a possible (or not) signal for this broad resonance. We discuss the production of this elusive meson in peripheral heavy-ion collisions. However, we focus attention on the observation of its $\gamma \gamma$ decay. We believe that the observation of this decay could settle definitively the existence of this particle as well as of its quark (or gluon) content. Although its branching ratio into photons is quite small compared to the main one $(\pi \pi)$, we will argue that the high photon luminosity in peripheral heavy-ion collisions may allow its observation, as long as it has the very same properties predicted in Ref. [7].

Photons with invariant mass of $\mathrm{O}(1 \mathrm{GeV})$ are also one possible signal for the quark-gluon plasma, therefore the detectors at RHIC have high efficiency to detect these photons, which is a welcomed capability in regard to the final states that we discuss in this work. The background for the twophoton initiated process were discussed by Nystrand and Klein [8]. Double-Pomeron exchange may be a background source [9]. However, they are negligible after imposing the peripheral condition $b>2 R_{A}$ as long as we operate with very heavy ions [10]. The cuts to remove the background are discussed in Ref. [8], i.e., the final state must have (i) low multiplicity, (ii) small summed transverse momentum, and (iii) be centered around rapidity 0 with a fairly narrow width

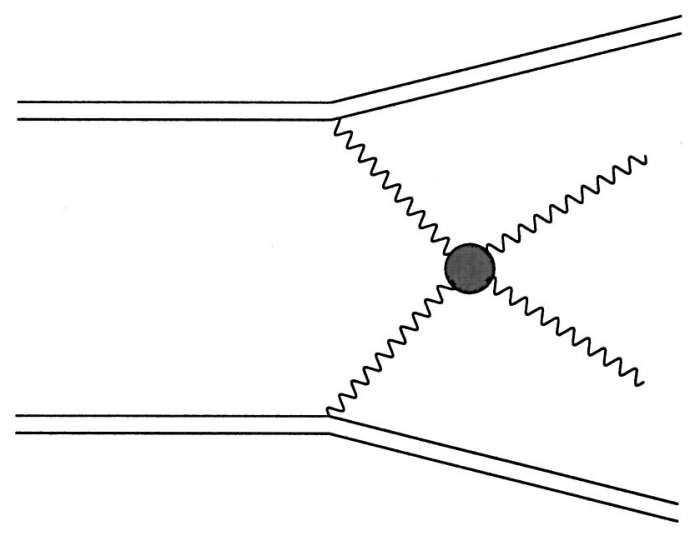

FIG. 1. Diagram for $\gamma \gamma$ fusion in a peripheral heavy-ion collision. The blob represents a continuum or resonant process leading to a two-photon final state. 
of center-of-mass rapidity $\left(y_{\text {c.m. }} \lesssim 1-2\right)$. These cuts are easily implemented when we analyze the reaction proposed in this work: $Z Z \rightarrow Z Z \gamma \gamma$.

The remainder of the paper is organized as follows. In the second section we introduce the photon distribution in the ion that will be used in our calculations. Section III contains the discussion of the $\gamma \gamma \rightarrow \gamma \gamma$ process and the cuts that we will adopt for this reaction. In Sec. IV we present values for the production of resonances made of quarks (or gluons), and Sec. V contains a discussion about the possibility of observing the $\sigma$ meson in its two photon decay. The last section contains our conclusions.

\section{PHOTON DISTRIBUTION IN THE ION}

The photon distribution in the nucleus can be described using the equivalent-photon or Weizsäcker-Williams approximation in the impact parameter space. Denoting by $F(x) d x$ the number of photons carrying a fraction between $x$ and $x+d x$ of the total momentum of a nucleus of charge $Z e$, we can define the two-photon luminosity through

$$
\frac{d L}{d \tau}=\int_{\tau}^{1} \frac{d x}{x} F(x) F(\tau / x),
$$

where $\tau=\hat{s} / s, \hat{s}$ is the square of the center of mass system (c.m.s.) energy of the two photons and $s$ of the ion-ion system. The total cross section of the process $Z Z \rightarrow Z Z \gamma \gamma$ is

$$
\sigma(s)=\int d \tau \frac{d L}{d \tau} \hat{\sigma}(\hat{s}),
$$

where $\hat{\sigma}(\hat{s})$ is the cross section of the subprocess $\gamma \gamma \rightarrow \gamma \gamma$.

There remains only to determine $F(x)$. In the literature there are several approaches for doing so, and we choose the conservative and more realistic photon distribution of Ref. [3]. Cahn and Jackson [3], using a prescription proposed by Baur [2], obtained a photon distribution which is not factorizable. However, they were able to give a fit for the differential luminosity which is quite useful in practical calculations:

$$
\frac{d L}{d \tau}=\left(\frac{Z^{2} \alpha}{\pi}\right)^{2} \frac{16}{3 \tau} \xi(z),
$$

where $z=2 M R \sqrt{\tau}, M$ is the nucleus mass, $R$ its radius, and $\xi(z)$ is given by

$$
\xi(z)=\sum_{i=1}^{3} A_{i} e^{-b_{i} z}
$$

which is a fit resulting from the numerical integration of the photon distribution, accurate to $2 \%$ or better for $0.05<z$ $<5.0$, and where $A_{1}=1.909, A_{2}=12.35, A_{3}=46.28, b_{1}$ $=2.566, b_{2}=4.948$, and $b_{3}=15.21$. For $z<0.05$ we use the expression (see Ref. [3])

$$
\frac{d L}{d \tau}=\left(\frac{Z^{2} \alpha}{\pi}\right)^{2} \frac{16}{3 \tau}\left[\ln \left(\frac{1.234}{z}\right)\right]^{3}
$$

The condition for realistic peripheral collisions $\left(b_{\min }>R_{1}\right.$ $+R_{2}$ ) is present in the photon distributions shown above.

The two photon production in heavy ions collisions can also be intermediated by the diffractive subprocess $P P$ $\rightarrow \gamma \gamma$, where $P$ is the Pomeron. But, as was shown by some of us in Ref. [10], when the cut in the impact parameter is introduced the double-Pomerom exchange is not important for very heavy nuclei.

\section{THE CONTINUUM REACTION $\gamma+\gamma \rightarrow \gamma+\gamma$}

The reaction $\gamma \gamma \rightarrow \gamma \gamma$ (given by the fermion box diagram) was first calculated exactly by Karplus and Neuman using the usual Feynman techniques [11]. Some time later this process was calculated by De Tollis using the Mandelstam representation and dispersion relations [12]. They encountered relatively simple helicity amplitudes for this process. Of the 16 helicity amplitudes, due to symmetry properties, the number of independent amplitudes will be only $5[13,14]$, that may be chosen to be $M_{++++}$, $M_{++--}, M_{+-+-}, M_{+--+}$, and $M_{+++-}$, where the + or - denotes the circular polarization values +1 and -1 . The remaining helicity amplitudes may be obtained from parity and permutation symmetry.

Of these five helicity amplitudes, three are related by crossing, hence it is sufficient to give just three, that in agreement with Refs. [12,14] are

$$
\begin{aligned}
& M_{++++}=1+\left\{2+\frac{4 s_{t}}{r_{t}}\right\} B\left(s_{t}\right)+\left\{2+\frac{4 t_{t}}{r_{t}}\right\} B\left(t_{t}\right) \\
& +\left\{\frac{2\left(s_{t}^{2}+t_{t}^{2}\right)}{r_{t}^{2}}-\frac{2}{r_{t}}\right\}\left[T\left(s_{t}\right)+T\left(t_{t}\right)\right]+\left\{-\frac{1}{s_{t}}\right. \\
& \left.+\frac{1}{2 r_{t} s_{t}}\right\} I\left(r_{t}, s_{t}\right)+\left\{-\frac{1}{t_{t}}+\frac{1}{2 r_{t} t_{t}}\right\} I\left(r_{t}, t_{t}\right) \\
& +\left\{-\frac{2\left(s_{t}^{2}+t_{t}^{2}\right)}{r_{t}^{2}}+\frac{4}{r_{t}}+\frac{1}{s_{t}}+\frac{1}{t_{t}}+\frac{1}{2 s_{t} t_{t}}\right\} I\left(s_{t}, t_{t}\right), \\
& M_{+++-}=-1+\left\{-\frac{1}{r_{t}}-\frac{1}{s_{t}}-\frac{1}{t_{t}}\right\}\left[T\left(r_{t}\right)+T\left(s_{t}\right)+T\left(t_{t}\right)\right] \\
& +\left\{\frac{1}{t_{t}}+\frac{1}{2 r_{t} s_{t}}\right\} I\left(r_{t}, s_{t}\right)+\left\{\frac{1}{s_{t}}+\frac{1}{2 r_{t} s_{t}}\right\} I\left(r_{t}, s_{t}\right) \\
& +\left\{\frac{1}{r_{t}}+\frac{1}{2 s_{t} t_{t}}\right\} I\left(s_{t}, t_{t}\right), \\
& M_{++--}=-1+\frac{1}{2 r_{t} s_{t}} I\left(r_{t}, s_{t}\right)+\frac{1}{2 r_{t} s t_{t}} I\left(r_{t}, t_{t}\right) \\
& +\frac{1}{2 s_{t} t_{t}} I\left(s_{t}, t_{t}\right)
\end{aligned}
$$

where $r_{t}, s_{t}$, and $t_{t}$ are related with the standard Mandelstam variables $s, t$, and $u$ by 


$$
r_{t}=\frac{1}{4} \frac{s}{m_{f}^{2}}, \quad s_{t}=\frac{1}{4} \frac{t}{m_{f}^{2}}, \quad t_{t}=\frac{1}{4} \frac{u}{m_{f}^{2}} .
$$

$m_{f}$ is the mass of the fermion in the loop.

The remaining 13 amplitudes may be obtained from these by using the relations

$$
\begin{gathered}
M_{+++-}=M_{++-+}=M_{+-++}=M_{-+++}, \\
M_{+-+-}(s, t, u)=M_{++++}(u, t, s), \\
M_{+--+}(s, t, u)=M_{++++}(t, s, u),
\end{gathered}
$$

where $s, t$, and $u$ are the Mandelstam variables.

The transcendental functions $B, T$, and $I$ that appear in Eq. (6) were defined by Karplus and Neuman [11], and are

$$
\begin{aligned}
B(x) & =\sqrt{1-\frac{1}{x}} \operatorname{arcsinh}(\sqrt{-x})-1, \quad(x<0) \\
& =\sqrt{1-\frac{1}{x}} \arcsin (\sqrt{x})-1, \quad(0<x<1) \\
& =\sqrt{1-\frac{1}{x}} \operatorname{arccosh}(\sqrt{x})-1-\frac{\pi i}{2} \sqrt{1-\frac{1}{x}}, \quad(1<x),
\end{aligned}
$$

$$
\begin{aligned}
T(x) & =[\operatorname{arcsinh}(\sqrt{-x})]^{2}, \quad(x<0) \\
& =-[\arcsin (\sqrt{x})]^{2}, \quad(0<x<1) \\
& =[\operatorname{arccosh}(\sqrt{x})]^{2}-\frac{\pi^{2}}{4}-i \pi \operatorname{arcosh}(\sqrt{x}), \quad(1<x),
\end{aligned}
$$

$$
\begin{gathered}
I(x, y)=I(y, x), \\
\operatorname{Re}\{I(x, y)\}=\frac{1}{2 a} \operatorname{Re}\left\{\Phi\left(\frac{a+1}{a+b(x)}\right)+\Phi\left(\frac{a+1}{a-b(x)}\right)\right. \\
\left.-\Phi\left(\frac{a-1}{a+b(x)}\right)-\Phi\left(\frac{a-1}{a-b(x)}\right)+(x \leftrightarrow y)\right\} \\
\operatorname{Im}\{I(x, y)\}=-\frac{\pi}{2 a} \ln y[a+b(x)]^{2}, \quad(x \geqslant 1) \\
=-\frac{\pi}{2 a} \ln x[a+b(y)]^{2}, \quad(y \geqslant 1), \quad(10)
\end{gathered}
$$

where $a=\sqrt{1-(x+y) / x y}, x, y=r_{t}, s_{t}$, and $t_{t}$, and

$$
\begin{aligned}
b(x) & =\sqrt{1-\left(\frac{1}{x}\right)}, \quad \text { when } \quad(x<0) \quad \text { or } \quad(x>1) \\
& =i \sqrt{1-\left(\frac{1}{x}\right)}, \quad \text { when } \quad(0<x<1) .
\end{aligned}
$$

The Spence function, $\Phi(z)$, is defined as $\Phi(z)=\int_{0}^{z} \ln (1$ $-t) d t / t$, and its properties can been found in Ref. [15].

The differential cross section of photon pair production from photon fusion, i.e., the box diagram, is

$$
\frac{d \sigma}{d \cos \theta}=\frac{1}{2 \pi} \frac{\alpha^{4}}{s}\left(\sum_{f} q_{f}^{2}\right)^{4} \sum|M|^{2} .
$$

$\theta$ is the scattering angle, $\alpha$ is the fine-structure constant, $q_{f}$ is the charge of the fermion in the loop and the sum is over the leptons $e$ and $\mu$ and the quarks $u, d$, and $s$, which are the relevant particles in the mass scale that we shall discuss. Another possible contribution to this continuum process comes from pion loops, which, apart from possible double counting, were shown to be negligible compared to the above one [16]. The second sum is over the 16 helicity amplitudes, $M_{\lambda_{1} \lambda_{2} \lambda_{3} \lambda_{4}}$, where $\lambda_{1}$ and $\lambda_{2}$ correspond to polarizations of incoming photons and $\lambda_{3}$ and $\lambda_{4}$ for the outgoing photons. The matrix elements sumed over the final polarizations and averaged over the initial polarizations is given by

$$
\begin{aligned}
\sum|M|^{2}= & \frac{1}{2}\left\{\left|M_{++++}\right|^{2}+\left|M_{++--}\right|^{2}+\left|M_{+-+-}\right|^{2}\right. \\
& \left.+\left|M_{+--+}\right|^{2}+4\left|M_{+++-}\right|^{2}\right\} .
\end{aligned}
$$

We consider the scattering of light by light, that is, the reaction $\gamma \gamma \rightarrow \gamma \gamma$, in $\mathrm{Au}-\mathrm{Au}$ collisions for energies available at RHIC, $\sqrt{s}=200 \mathrm{GeV} /$ nucleon. We checked our numerical code reproducing the many results of the literature for the box subprocess, including asymptotic expressions for the low and high energies compared to the fermion mass present in the loop, and the peak value of the cross section (see, for instance, Ref. [13]).

In Fig. 2 the dependence of the ion-ion cross section with the cosine of the scattering angle $\theta$, in the two photon centerof-mass system, is shown for an invariant photon pair mass equal to $500 \mathrm{MeV}$. It is possible to observe that the cross section is strongly peaked in the backward direction, but is relatively flat out to $\cos \theta \approx 0.4$, where it starts rising very fast. It is symmetric with respect to $\theta$ and the same behavior is present in the forward direction.

The photon pair production in ion collisions over an invariant mass range between 300 and $1000 \mathrm{MeV}$ for different angular cuts is shown in Fig. 3. It is possible to observe that the cross sections is strongly restricted when the angular cut is more drastic, the difference between the cuts $10<\theta$ $<170^{\circ}$ and $60<\theta<120^{\circ}$ is quite large, the cross section is strongly suppressed for the second range. We will impose in all the calculations throughout this work a cut in the scattering angle equal to $|\cos \theta|=0.5$. This cut is conservative, but it will make it possible to compare the cross section of the box diagram with rival processes, that will be discussed in the following sections, as well as it is enough to eliminate the effect of double bremsstrahlung (which dominates the region of $|\cos \theta| \approx 1$ ). Finally, this kind of cut is totally consistent with the requirements proposed in Ref. [8]

The fermions that contribute in the box diagram are the leptons $e$ and $\mu$ and the quarks $u, d$, and $s$, which are impor- 


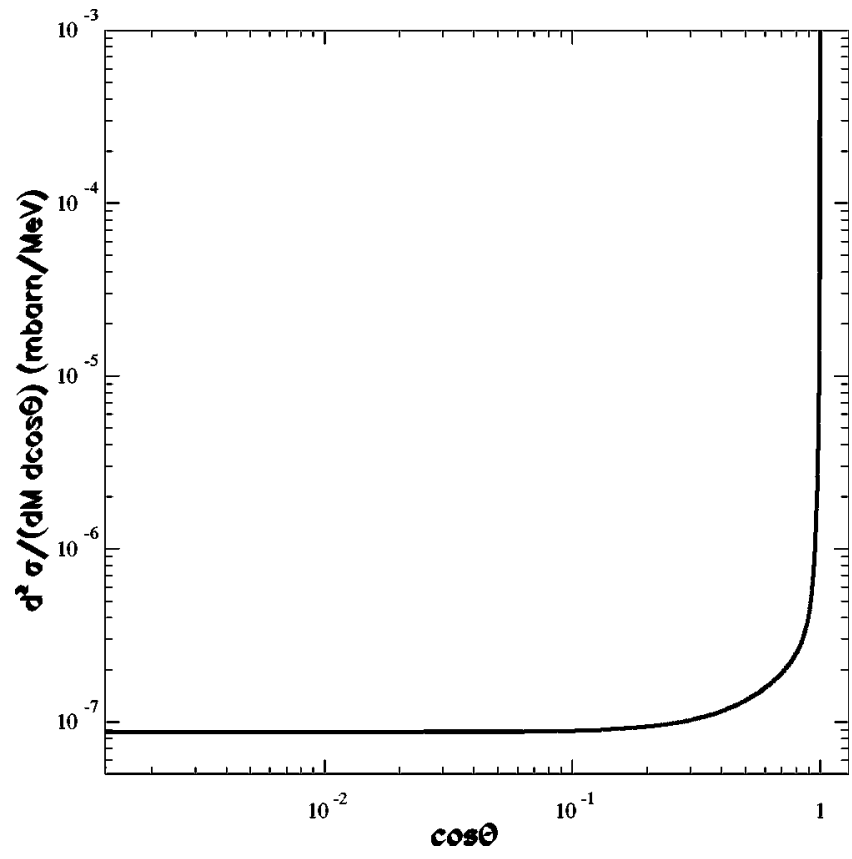

FIG. 2. Angular distribution of $Z Z \rightarrow Z Z \gamma \gamma$ scattering at an invariant mass of $500 \mathrm{MeV}$. The scattering angle $\theta$ is in the photon pair center-of-mass system.

tant for the mass range that we are interested (heavier quarks will give insignificant contributions and the same is true for the charged weak bosons). We assumed for their masses the following values: $m_{e}=0.5109 \mathrm{MeV}, m_{\mu}=105.6584 \mathrm{MeV}$, $m_{u}=5 \mathrm{MeV}, m_{d}=9 \mathrm{MeV}$, and $m_{s}=170 \mathrm{MeV}$.

The electron gives the major contribution to the total re-

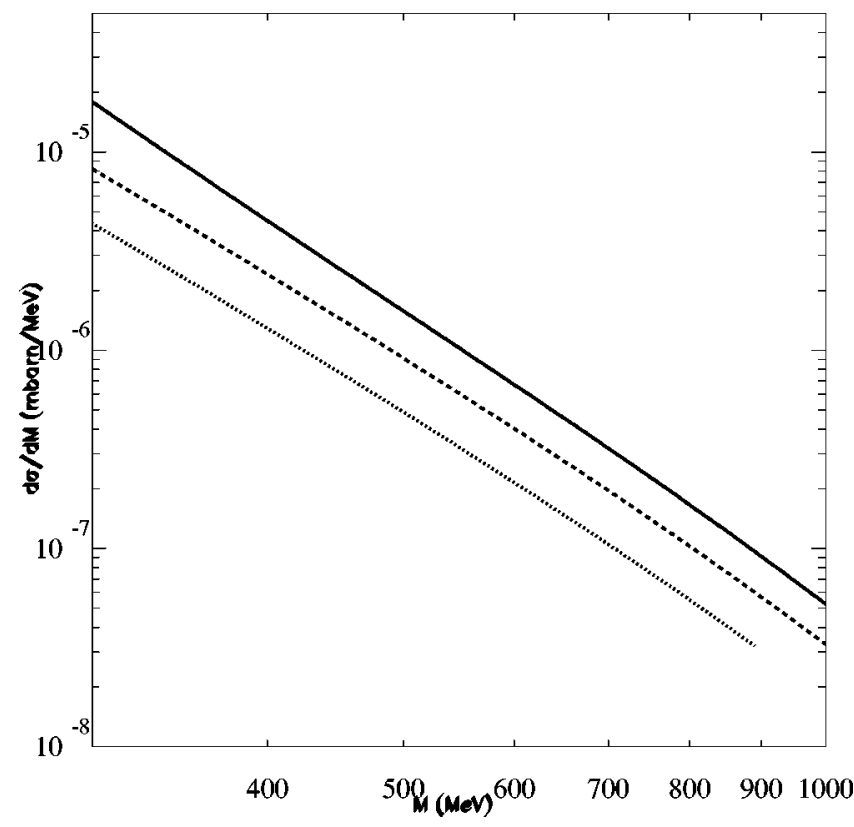

FIG. 3. Invariant mass distribution of photon pair production at RHIC via photon fusion through a fermion loop. Each curve represents a different cut in the scattering angle $\theta$, the solid line is for $10<\theta<170^{\circ}$, the dashed is for $40<\theta<140^{\circ}$, and the dotted one for $60<\theta<120^{\circ}$.

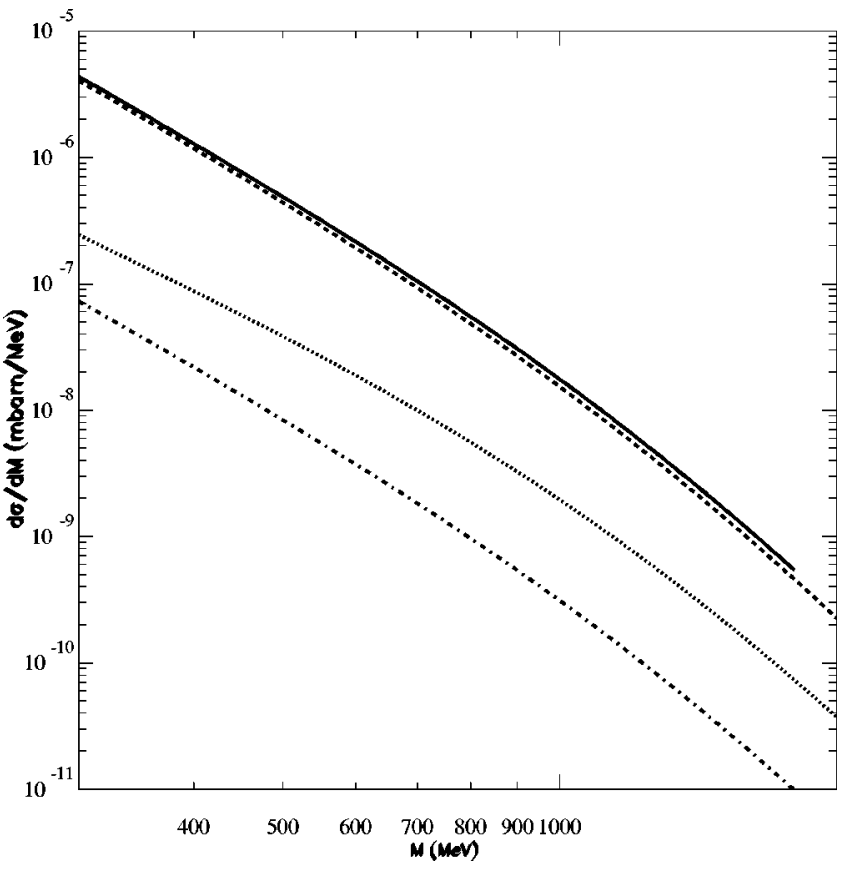

FIG. 4. Invariant mass distribution of photon pair production at RHIC center-of-mass energy of $\sqrt{s}=200 \mathrm{GeV}$ for each fermion loop. The solid line denotes the differential cross section for all fermions, the dashed one is the electron loop contribution, the dotted is the muon one, the dash-dotted is the $u$ quark contribution. The result is obtained with the cut $|\cos \theta|<0.5$.

sult as can be seen in Fig. 4. The second most important contribution is due to the muon, but it is at least one order of magnitude smaller than the electron one. The $d$ and $s$ quark contribution [up to $\mathrm{O}(2 \mathrm{GeV})]$ are smaller due to their masses and charges, because the process is proportional to $\left(q_{f}^{2}\right)^{4}$ where $q_{f}$ is their charge. Their contribution is also insignificant compared to the electron result.

As discussed in Ref. [4] the $\gamma \gamma$ scattering can indeed be measured in peripheral heavy ion collisions. The cut in the angular distribution gives back to back photons in the central rapidity region free of the background. However, as we shall see in the next section, there are gaps where the $\gamma \gamma \rightarrow \gamma \gamma$ process is overwhelmed by the presence of resonances like the $\eta, \eta^{\prime}$, and others. Even the broad $\sigma$ resonance could be of the order of the continuum process. Just to give one idea of the number of events, with a luminosity of 2.0 $\times 10^{26} \mathrm{~cm}^{-2} \mathrm{~s}^{-1}$ [8] and choosing a bin of energy of 200 $\mathrm{MeV}$, centered at the energy of $700 \mathrm{MeV}$ (which is free of any strong resonance decaying into two-photons), we have 1532 events/year assuming $100 \%$ efficiency in the tagging of the ions and photon detection.

\section{RESONANCES DECAYING INTO TWO-PHOTONS}

Photon pair production via the box diagram is a background to $\gamma \gamma \rightarrow R \rightarrow \gamma \gamma$ process (or vice versa), both have the same initial and final states, and for this reason they can interfere one in another. Normally the interference between a resonance and a continuum process is unimportant, because on resonance the two are out of phase. 


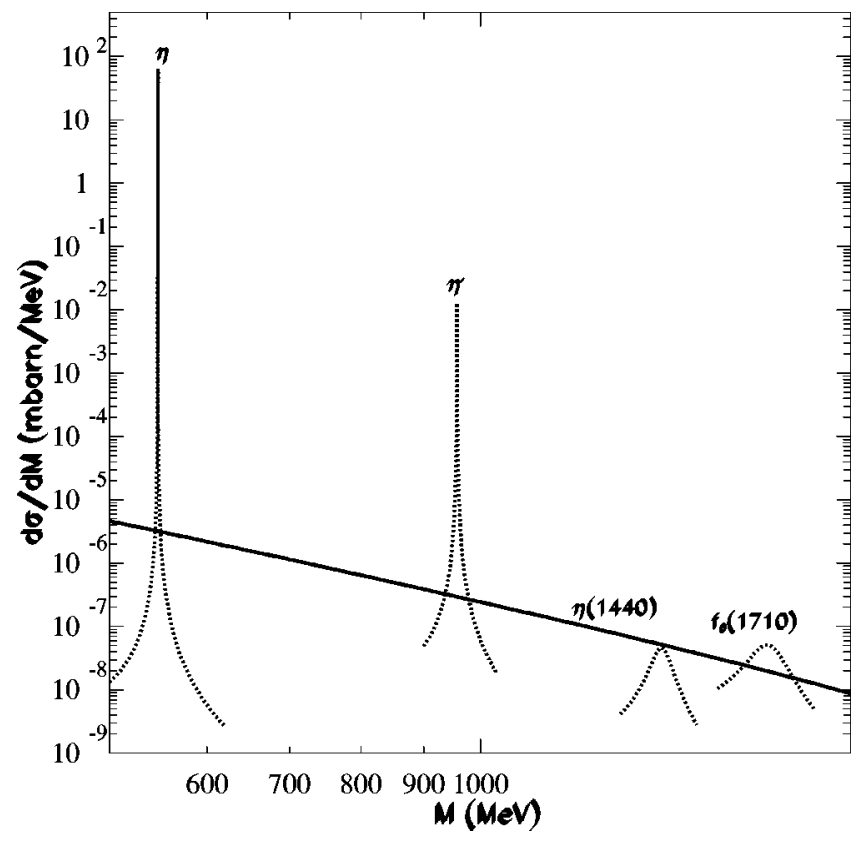

FIG. 5. Invariant mass distribution of photon production (with the cut $|\cos \theta|<0.5$ ). The solid curve is for the box diagram, the dashed curves are due to the process $\gamma \gamma \rightarrow R \rightarrow \gamma \gamma$, where $R$ are the pseudoscalars resonances $\eta$ and $\eta^{\prime}$ and the glueballs candidates $\eta(1410)$ and $f_{0}(1710)$.

The total cross section for the elementary subprocess $\gamma \gamma$ $\rightarrow R \rightarrow \gamma \gamma$ assuming a Breit-Wigner profile is

$$
\frac{d \sigma_{Z Z}^{\gamma \gamma}}{d M}=16 \pi \frac{d L}{d M} \frac{\Gamma^{2}(R \rightarrow \gamma \gamma)}{\left(M^{2}-m_{R}^{2}\right)^{2}+m_{R}^{2} \Gamma_{\text {total }}^{2}},
$$

where $M$ is the energy of the photons created by the collision of the ions. $\Gamma(R \rightarrow \gamma \gamma)\left(\equiv \Gamma_{\gamma \gamma}\right)$ and $\Gamma_{\text {total }}$ are the partial and total decay width of the resonance with mass $m_{R}$ in its rest frame.

We are going to discuss only $J=0$ resonances made of quarks as well of gluons. The reaction $\gamma \gamma \rightarrow \pi^{0} \rightarrow \gamma \gamma$ was already discussed many years ago [17], where it was claimed that the interference vanishes. This result was criticized by De Tollis and Violini [16], affirming (correctly) that the interference exists. However, as we will discuss afterwards, off resonance the interference is negligible. If the interference is neglected, Eq. (13) can be used and we show in Fig. 5 the result for some resonance production $\left[\eta, \eta^{\prime}, \eta(1440), f_{0}(1710)\right]$, whose invariant mass of the produced photon pair is between 500 and $2000 \mathrm{MeV}$. For comparison we also show the curve of the continuum process. It is possible to see in that figure the well pronounced peaks of the resonances $\eta$ and $\eta^{\prime}$. We assumed for their masses the values of 547.3 and $957.78 \mathrm{MeV}$, respectively, the $\eta$ total decay width is equal to $1.18 \mathrm{keV}$ and the $\eta^{\prime}$ one is equal to $0.203 \mathrm{MeV}$. Their partial decay width into photons are 0.46 $\mathrm{keV}(\eta)$ and $4.06 \mathrm{keV}\left(\eta^{\prime}\right)$.

In the same figure we can see the predicted cross section for the glueball candidates production in peripheral heavy ion collisions by double photon fusion.
We restrict the analysis to the $J=0$ glueballs candidates $\eta(1440)$ and $f_{0}(1710)$. For the $\eta(1440)$ we used the mass and total decay width values of Ref. [18], $m_{R}$ $=1405 \mathrm{MeV}, \Gamma_{\text {total }}=56 \mathrm{MeV}$, for the decay width into photons we use the value given in Ref. [19], $\Gamma_{\gamma \gamma}$ $=5.4 \mathrm{keV}$. We see in Fig. 5 that the peak for this resonance is of the same order of the continuum process. For the other glueball candidate, $f_{0}(1710)$, the peak is clearly above the background. For this one we assumed the values listed in Ref. [18] of mass and total width, $m_{R}=1715 \mathrm{MeV}, \Gamma_{\text {total }}$ $=125 \mathrm{MeV}$, and for the two-photon decay width we adopted the value encountered by the ALEPH Collaboration [20], $\Gamma_{\gamma \gamma}=21.25 \mathrm{keV}$. In all these cases the resonances can be easily studied in peripheral heavy ion collisions.

Off resonance we can expect a negligible contribution for the process $\gamma \gamma \rightarrow R \rightarrow \gamma \gamma$ and consequently the same for its interference with the continuum process. However, it is instructive to present a more detailed argument about why the interference can be neglected. In order to do so we are obliged to introduce a model to calculate the amplitudes for the process $\gamma \gamma \rightarrow R \rightarrow \gamma \gamma$. These amplitudes will be computed with the help of an effective Lagrangian for the pseudoscalar interaction with photons (the scalar case will be discussed in the next section), which is given by $g_{p} \varepsilon_{\lambda \mu \nu \rho} F^{\lambda \mu} F^{\nu \rho} \Phi_{p}$, where $g_{p}$ is the coupling of the photons to the pseudoscalar field $\Phi_{p}, \varepsilon_{\lambda \mu \nu \rho}$ is the antisymmetric tensor, and $F^{\lambda \mu}$ is the electromagnetic field four-tensor.

The amplitudes for $\gamma \gamma \rightarrow \gamma \gamma$ intermediated by a pseudoscalar hadronic resonance are [16]

$$
\begin{gathered}
M_{++++}=\frac{2 \pi}{\alpha^{2}} F\left(\lambda r_{t}\right), \\
M_{+-+-}=\frac{2 \pi}{\alpha^{2}} F\left(\lambda t_{t}\right), \\
M_{+--+}=\frac{2 \pi}{\alpha^{2}} F\left(\lambda s_{t}\right), \\
M_{+++-}=0, \\
M_{++--}=-\frac{2 \pi}{\alpha^{2}}\left\{F\left(\lambda s_{t}\right)+F\left(\lambda t_{t}\right)+F\left(\lambda r_{t}\right)\right\},
\end{gathered}
$$

where $\alpha$ is the fine-structure constant, $\lambda=\left(m_{f} / m_{R}\right)^{2}$, and

$$
F(x)=16 x^{2} \frac{\Gamma_{\gamma \gamma}}{m_{R}}\left(4 x-1+i \frac{\Gamma_{\text {total }}}{m_{R}}\right)^{-1} .
$$

The presence of the fine-structure constant in Eq. (14) is a consequence of the fact that the amplitudes $M$ in these equations will be used in Eq. (12), so it is necessary to get the correct dependence of the partial cross section with this constant.

A numerical evaluation of the cross section using Eq. (14) (for the same resonances present in Fig. 5) shows a totally negligible effect off resonance in comparison with the box 
TABLE I. Number of events/year above background for the $\eta$, $\eta^{\prime}$, and $f_{0}(1710)$ resonances.

\begin{tabular}{lc}
\hline \hline Particle & Events/year \\
\hline$\eta$ & $7.44 \times 10^{5}$ \\
$\eta^{\prime}$ & $2.67 \times 10^{4}$ \\
$f_{0}(1710)$ & 42 \\
\hline \hline
\end{tabular}

contribution. On resonance the two processes are out of phase and the interference is absent. We can now proceed with an argument showing that the interference is not important. Let us assume that off resonance the processes are in phase, and for a moment we forget the $t$ and $u$ channels contribution in Eq. (14). The $s$ channel contribution can be written as $M_{2} /\left(s-m_{R}^{2}+i \Gamma_{R} m_{R}\right)$, and denoting the continuum contribution by $M_{1}$ we can write the following interference term:

$$
\begin{aligned}
& 2 \frac{s}{\left(s-m_{R}^{2}\right)^{2}+\Gamma_{R}^{2} m_{R}^{2}}\left[\left(\operatorname{Re} M_{1} \operatorname{Re} M_{2}+\operatorname{Im} M_{1} \operatorname{Im} M_{2}\right)\left(s-m_{R}^{2}\right)\right. \\
& \left.\quad+\left(\operatorname{Re} M_{1} \operatorname{Im} M_{2}-\operatorname{Im} M_{1} \operatorname{Re} M_{2}\right) \Gamma_{R} m_{R}\right]
\end{aligned}
$$

We can verify that the term proportional to $s-m_{R}^{2}$ integrates to zero when integrated in a bin centered at $m_{R}^{2}$. With the second term the situation is different. If $\operatorname{Im} M_{1}$ or $\operatorname{Im} M_{2}$ $\neq 0$ (assuming $\operatorname{Re} M_{1}$ and $\operatorname{Re} M_{2} \neq 0$ ) then there is a nontrivial interference. However, since we are dealing with $J$ $=0$ amplitudes, the only nonvanishing helicity amplitudes are those in which the initial helicities and the final helicities are equal. Inspection of the $M_{++++}$and $M_{++--}$amplitudes of the box diagram (in the limit $m_{f} \ll m_{R}$ ) shows that they are purely real, and the same happens with $M_{2}$ [obtained from the $s$ channel contribution of Eq. (14)], resulting in a vanishing interference. Of course, this analysis is model dependent. In particular, at the quark level the coupling $g_{p}$ has to be substituted by a triangle diagram, which may have a real as well as an imaginary part (see, for instance, Ref. [21]). However, this coupling is real for heavy quarks and its imaginary part is quite suppressed if the resonance couples mostly to light quarks $\left(m_{f} \ll m_{R}\right.$, what happens for the resonances that we are considering in the case of the $u$ and $d$ quarks, for the $s$ quark the suppresion is not so strong in the $\eta$ case, but we still have an extra suppression due to its electric charge). Finally, the interference does appear when we consider the amplitudes with the resonance exchanged in the $t$ and $u$ channels, but it is easy to see that they are kinematically suppressed and also proportional to the small value of the total decay width. If the total width is large (as we shall discuss in the next section) the interference cannot be neglected. Although the above argument has to rely on models for the low energy hadronic physics, we believe that the direct comparison between the resonant and the continuum processes, as presented in Fig. 5, is fairly representative of the actual result.

In Table I we show the number of events above background for the $\eta, \eta^{\prime}$, and $f_{0}(1710)$ which, as shown in Fig.
5 are clearly above the box contribution. In the case of the $f_{0}(1710)$, as well as in the $\sigma$ meson case to be discussed in the next section, the decay of the resonance into a pair of neutral pions is present. A pair of neutral pions can also be produced in a continuous two-photon fusion process. The rates for all the reactions discussed in this work can be modified if both neutral pions, no matter if they come from a resonance or a continuos process, are misidentified with photons. This accidental background can be easily isolated measuring its invariant mass distribution, and making a cut that discriminates a single photon coming from the processes that we are studying, from one that produced two neutral mesons (mostly pions) subsequently decaying into two photons. For example, in the case of the sigma meson (see next section) each one of the neutral pions from its much larger hadronic decay should be misidentified. These pions would produce pairs of photons with a large opening angle $\phi$, where $\cos (\phi / 2)=\sqrt{1-4 m_{\pi}^{2} / m_{\sigma}^{2}}$. However, the calorimeters already in use in many experiments make it possible to distinguish between these two- and single-photon events with high efficiency (see, for instance, Ref. [22]).

There is also another accidental background which may cause problems to the measurement of the resonance decay into photons. This is the contribution from $\gamma$-Pomeron $\rightarrow \mathrm{V}$ $\rightarrow \gamma \gamma X$. The vector mesons, $\mathrm{V}$, are produced with a $p_{T}$ distribution similar to the resonance production and at higher rates than some of the processes $\gamma \gamma \rightarrow R$, e.g., $\gamma$-Pomeron $\rightarrow \omega$ rate is $10 \mathrm{~Hz}$ at RHIC [23], three orders of magnitude higher than for a similar mass meson of spin 0 or 2 . The $\omega$ branching ratio to three photons is $8.5 \%$. If a small $p_{T}$ photon from this decay is undetected, one is left with a low $p_{T}$ two-photon final state that could be taken for a lighter resonance. At higher masses, one also has $\phi \rightarrow \eta \gamma, \pi^{0} \gamma, K_{L} K_{S}$ $\rightarrow \gamma X$ as well as $\gamma \gamma \rightarrow f_{2}(1270) \rightarrow \pi^{0} \pi^{0}$ and possibly copious production of $\rho(1450)$ and $\rho(1700)$ by $\gamma$-Pomeron interactions. Clearly a full simulation of all these background processes is beyond the scope of this paper but should be kept in mind when measuring two-photon final states.

\section{CAN WE OBSERVE THE $\sigma$ MESON IN PERIPHERAL COLLISIONS?}

The possible existence of light scalar mesons (with masses less than about $1 \mathrm{GeV}$ ) has been a controversial subject for roughly 40 years. There are two aspects: the extraction of the scalar properties from experiment and their underlying quark substructure. Because the $J=0$ channels may contain strong competing contributions, such resonances may not necessarily dominate their amplitudes and could be hard to "observe." In such an instance their verification would be linked to the model used to describe them.

Part of the motivation to study the two-photon final states in peripheral heavy ion collisions was exactly to verify if we can observe such scalar mesons in its $\gamma \gamma$ decay. Although this decay mode is quite rare, it has the advantage of not being contaminated by the strong interaction of the hadronic final states. In particular, it may allow to investigate the possible existence of the sigma meson. This meson is expected to have a mass between 400 and $1200 \mathrm{MeV}$ and decay width 
between 300 and $500 \mathrm{MeV}$, decaying predominantly into two pions. Of course, another decay channel is into two photons, with the background discussed in Sec. III.

Recently the E791 Collaboration at Fermilab found a strong experimental evidence for a light and broad scalar resonance, that is, the sigma, in the $D^{+} \rightarrow \pi^{-} \pi^{+} \pi^{+}$decay [7]. The resonant amplitudes present in this decay were analyzed using the relativistic Breit-Wigner function given by

$$
B W=\frac{1}{m^{2}-m_{0}^{2}+i m_{0} \Gamma(m)},
$$

with

$$
\Gamma(m)=\Gamma_{0} \frac{m_{0}}{m}\left(\frac{p^{*}}{p_{0}^{*}}\right)^{2 J+1} \frac{{ }^{J} F^{2}\left(p^{*}\right)}{{ }^{J} F^{2}\left(p_{0}^{*}\right)},
$$

where $m$ is the invariant mass of the two photons forming a spin- $J$ resonance. The functions ${ }^{J} F$ are the Blatt-Weisskopf damping factors [24]: ${ }^{0} F=1$ for spin 0 particles, ${ }^{1} F$ $=1 / \sqrt{1+\left(r p^{*}\right)^{2}}$ for spin 1 and ${ }^{2} F$ $=1 / \sqrt{9+3\left(r p^{*}\right)^{2}+\left(r p^{*}\right)^{4}}$ for spin 2. The parameter $r$ is the radius of the resonance $(\approx 3 \mathrm{fm})[25]$ and $p^{*}=p^{*}(M)$ the momentum of decay particles at mass $M$, measured in the resonance rest frame, $p_{0}^{*}=p_{0}^{*}\left(m_{R}\right)$. The Dalitz plot of the decay can hardly be fitted without a $0^{++}(\sigma)$ resonance. The values of mass and total decay width found by the collaboration with this procedure are $478_{-23}^{+24} \pm 17 \mathrm{MeV}$ and $324_{-40}^{+42} \pm 21 \mathrm{MeV}$, respectively.

We will discuss if this resonance can be found in peripheral heavy ion collisions through the subprocess $\gamma \gamma \rightarrow \sigma$ $\rightarrow \gamma \gamma$. It is important to note that all the values related to the $\sigma$, like mass or partial widths, that can be found in the literature are very different and model dependent. In particular, we find the result of the E791 experiment very compelling and among all the possibilities we will restrict ourselves to their range of mass and total decay width, while we vary the partial width into two photons.

For the $\sigma$ decay width into two photons we assume the values obtained by Pennington and Boglione, $3.8 \pm 1.5 \mathrm{keV}$ and $4.7 \pm 1.5 \mathrm{keV}$ [26], and the value of $10 \pm 6 \mathrm{keV}$ [27]. The results obtained are in Fig. 6, the dashed curve is for $\Gamma_{\gamma \gamma}=3.8 \mathrm{keV}$, the dotted one is for $\Gamma_{\gamma \gamma}=4.7 \mathrm{keV}$ and the dot-dashed one is for $\Gamma_{\gamma \gamma}=10 \mathrm{keV}$. In all cases we used the mass and total decay width obtained by the E791 Collaboration, $m_{\sigma}=478 \mathrm{MeV}$ and $\Gamma_{\text {total }}=324 \mathrm{MeV}$. In the same figure it can be seen the curve due to the box diagram. In all cases we used the angular cut $-0.5<\cos \theta<0.5$.

It has been verified in the case of $\pi \pi$ scattering that the use of a constant total width in the $\sigma$ resonance shape is not a good approximation [28]. In our case we will discuss the $\gamma \gamma \rightarrow \gamma \gamma$ process above the two pions threshold where the peculiarities of the broad resonance, basically due to the $\sigma$ decay into pions, are not so important. Of course, another reason to stay above $300 \mathrm{MeV}$ is that we are also far from the pion contribution to $\gamma \gamma$ scattering. In any case we also computed the cross section with an energy dependent total width $\Gamma(m) \simeq \Gamma_{0}\left(p^{*} / p_{0}^{*}\right)^{2 J+1}$, which, as shown by Jackson

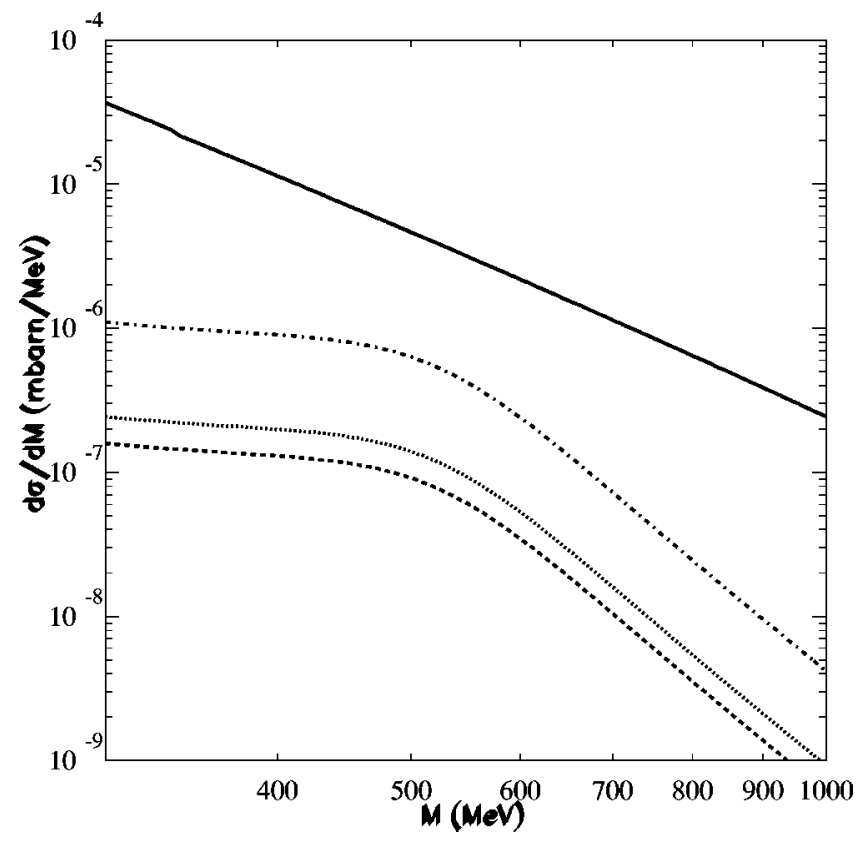

FIG. 6. Invariant mass distribution of photon production. The solid curve is the box diagram, the dashed curve is for the process $\gamma \gamma \rightarrow \sigma \rightarrow \gamma \gamma$ when $\Gamma_{\gamma \gamma}=10 \mathrm{keV}$, the dotted one is for $\Gamma_{\gamma \gamma}=4.7$ $\mathrm{keV}$, and the dot-dashed one is for $\Gamma_{\gamma \gamma}=3.8 \mathrm{keV}$. The angular cut is equal to $-0.5<\cos \theta<0.5$ in all curves.

many years ago [29], is more appropriate for a quite broad resonance. The net effect is a slight distortion of the cross section shape with a small increase of the total cross section. Since this one is a negligible effect compared to the one that we will present in the sequence we do not shall consider it again.

Up to now we assumed in this analysis only the BreitWigner profile for the $\sigma$ contribution and it is interesting to discuss the full process (including the $t$ and $u$ channels) within a given model to verify how good is our approximation.

To compute the amplitudes for $\gamma \gamma \rightarrow \gamma \gamma$ including the presence of a scalar resonance we can assume the interaction $g_{s} F^{\mu \nu} F_{\mu \nu} \Phi_{s}$, where $g_{s}$ is the coupling of the photons to the scalar field $\Phi_{s}$. These amplitudes are

$$
\begin{gathered}
M_{++++}=-\frac{2 \pi}{\alpha^{2}} F\left(\lambda r_{t}\right), \\
M_{+-+-}=-\frac{2 \pi}{\alpha^{2}} F\left(\lambda t_{t}\right), \\
M_{+--+}=-\frac{2 \pi}{\alpha^{2}} F\left(\lambda s_{t}\right), \\
M_{+++-}=0, \\
M_{++--}=-\frac{2 \pi}{\alpha^{2}}\left\{F\left(\lambda s_{t}\right)+F\left(\lambda t_{t}\right)+F\left(\lambda r_{t}\right)\right\} .
\end{gathered}
$$




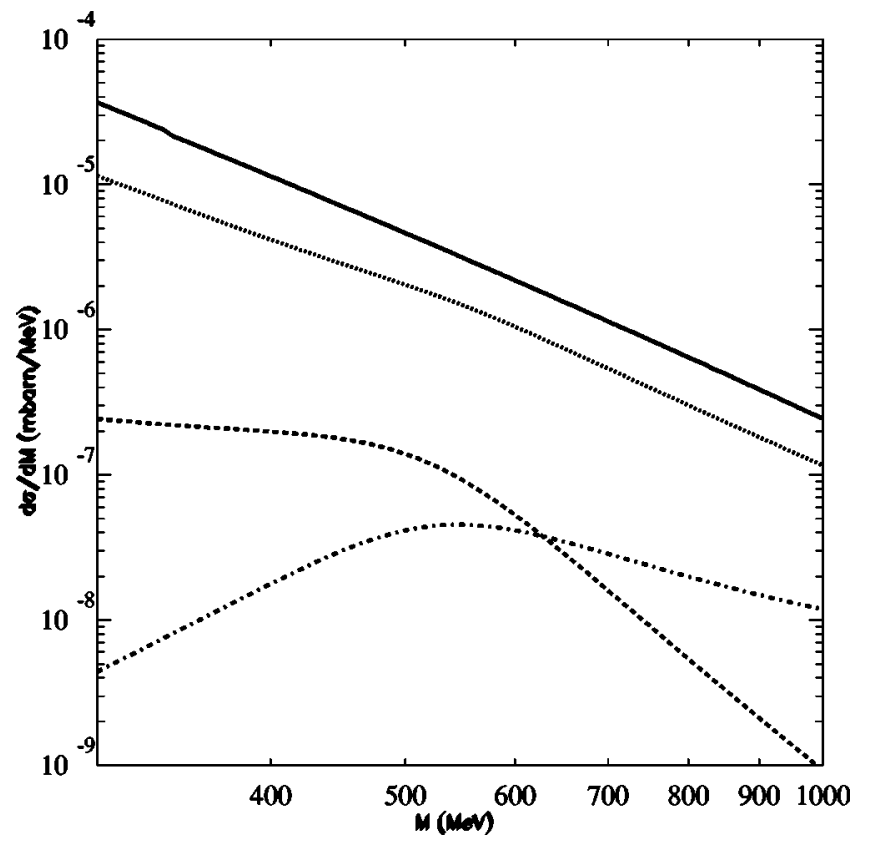

FIG. 7. Invariant mass distribution of photon pair production. The solid curve is due to box diagram only, the dashed one is due to the process $\gamma \gamma \rightarrow \sigma \rightarrow \gamma \gamma$ in the Breit-Wigner approximation, the dash-dotted is the scalar contribution of Eq. (16), and the dotted one is due to the total process, computed with box amplitudes plus the scalar boson amplitudes of Eq. (16). In all cases $\Gamma_{\gamma \gamma}=4.7 \mathrm{keV}$, and the angular cut is equal to $-0.5<\cos \theta<0.5$.

Note that this interaction violates unitarity and is appropriate only at energies up to the $\sigma$ mass.

In Fig. 7 we show the full cross section for the process $\gamma \gamma \rightarrow \gamma \gamma$ due to the box diagram and the existence of the scalar resonance $\sigma$ with amplitudes given by Eq. (16), computed with $\Gamma_{\gamma \gamma}=4.7 \mathrm{keV}$. Contrarily to the previous cases the $\sigma$ is a quite large "resonance" and due to its large decay width off resonance the interference is not negligible (the argument of the previous section obviouly fails). Moreover, the interference is destructive. In Fig. 7 we can see the continuous curve which is due only to the box diagram, the dashed one is the cross section of a scalar resonance computed with a Breit-Wigner profile, Eq. (13), the dash-dotted curve is due only to the scalar contribution of Eq. (16), and the dotted one is obtained when we consider the total cross section for two photon production in peripheral heavy ion collisions, i.e., the box diagram amplitudes plus the helicity amplitudes listed in Eq. (16). The figure shows clearly that the above mentioned interference is destructive.

There is an important point to comment about the above result. The effective Lagrangian model used to compute the $\sigma$ contribution to the photon pair production above $M$ $\approx 600 \mathrm{MeV}$ gives a larger contribution to the cross section than the Breit-Wigner one. We verified that the $t$ and $u$ channels produce small variations in the result obtained when we consider only the $s$ channel, but this one already grows as any nonunitary amplitude calculated in this approach, and it is unclear to us how far we can trust in this model. At small masses the cross sections also differ in their behavior with $s$.

There are possible improvements that we may think of

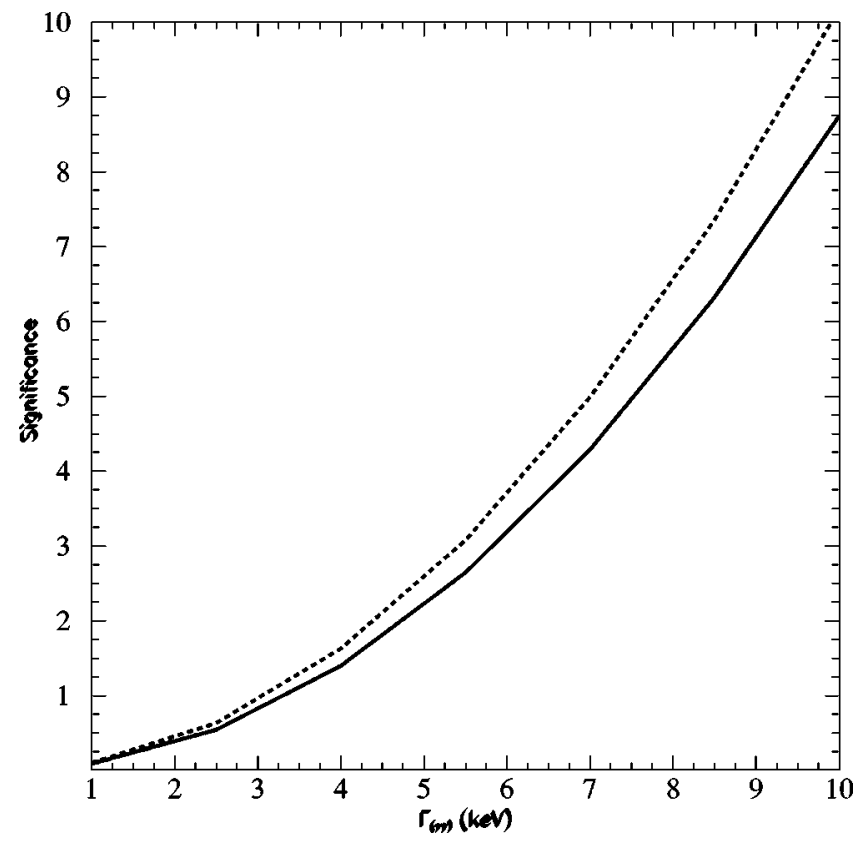

FIG. 8. Significance as a function of decay width into two photons, $\Gamma_{\gamma \gamma}$, for a sigma meson with mass equal to $478 \mathrm{MeV}$ and total decay width of $324 \mathrm{MeV}$. The solid curve was obtained integrating the cross sections in the interval $438<M<519 \mathrm{MeV}$, the dashed one in the interval $300<M<800 \mathrm{MeV}$.

towards a more reliable computation of the $\sigma$ meson exchange amplitudes, but all of them are going to be plagued by uncertainties as we remarked in the beginning of the section. Therefore we adopt the pessimistic view that the BreitWigner result is just a lower bound for our process, because effective Lagrangians tend to increase the contribution at large masses where the background vanishes rapidly, increasing the signal/background ratio. The Breit-Wigner profile does the opposite. The physical result is probably between both approaches. From the experimental point of view we would say that the reaction $\gamma \gamma \rightarrow \gamma \gamma$ has to be observed and any deviation from the continuum process must be carefully modeled until a final understanding comes out, with the advantage that the final state is not strongly interacting. Note that in this modelling the $\eta$ meson will contribute to $\gamma \gamma$ $\rightarrow \gamma \gamma$ in a small region of momentum, even so it has to be subtracted in order to extract the complete $\sigma$ signal.

We changed the values of the $\sigma$ mass and total width around the central ones reported by the E791 Collaboration. We do not observe large variations in our result, but noticed that it is quite sensitive to variations of the partial decay width into photons. It is interesting to look at the values of the significance which is written as $\mathcal{L} \sigma_{\text {signal }} / \sqrt{\mathcal{L} \sigma_{\text {back }}}$ and characterizes the statistical deviation of the number of the observed events from the predicted background. The significance as a function of the two-photon decay width of the sigma meson, with mass equal to $478 \mathrm{MeV}$ and total decay width of $324 \mathrm{MeV}$, is shown in Fig. 8, where we used a luminosity of $\mathcal{L}=2.0 \times 10^{26} \mathrm{~cm}^{-2} \mathrm{~s}^{-1}$ at RHIC and assumed one year of operation. The significance is above $2 \sigma 95 \%$ confidence level limit for two-photon decay width greater than $4.7 \mathrm{keV}$ while for a $5 \sigma$ discovery criteria can be ob- 
tained with $\Gamma_{\gamma \gamma}>7.5 \mathrm{keV}$. If $\Gamma_{\gamma \gamma}$ turns out to be smaller the numbers of Fig. 8 have to be scaled.

The numbers in Fig. 8 were computed with the signal given by the Breit-Wigner profile, and the background by the pure box diagram. The solid curve was obtained integrating the cross sections in the interval $438<M<519 \mathrm{MeV}$, corresponding to the interval of mass uncertainty, while the dashed curve resulted from the integration in the interval $300<M<800 \mathrm{MeV}$. Note that there is no reason, a priori, to restrict the measurement to a small bin of energy. This choice will depend heavily on the experimental conditions. When the signal is extracted from Eq. (16) and the cross sections are integrated in the larger interval (300-800 MeV), we obtain values for the significance more than one order of magnitude above the curves of Fig. 8, while the significance is below the curves of Fig. 8 up to a factor 10 when the cross sections are integrated in the smaller interval of energy. The Breit-Wigner profile probably gives an average of the results that could be obtained within different model calculations. Therefore for values of $\Gamma_{\gamma \gamma}$ already quoted in the literature the sigma meson has a chance to be seen in its two-photon decay mode. The discovery limits discussed above refer only to a statistical evaluation. Our work shows the importance of the complete simulation of the signal and background including an analysis of possible systematic errors that may decrease the significance.

\section{CONCLUSIONS}

Peripheral collisions at relativistic heavy ion colliders provide an arena for interesting studies of hadronic physics. One of the possibilities is the observation of light hadronic resonances, which will appear quite similarly to the twophoton hadronic physics at $e^{+} e^{-}$machines with the advantage of a huge photon luminosity peaked at small energies [1]. Due to this large photon luminosity it will become possible to discover resonances that couple weakly to the photons [5].

In this work we focus the attention at the peripheral reaction $Z Z \rightarrow Z Z \gamma \gamma$. This process is important because it may allow for the first time the observation of the continuous subprocess $\gamma \gamma \rightarrow \gamma \gamma$ in a complete collider physics environment. This possibility only arises due to enormous amount of photons carried by the ions at the RHIC energies.

The continuous subprocess is described by a Fermionic box diagram calculated many years ago. We computed the peripheral heavy ion production of a pair of photons, verify- ing which are the most important contributions to the loop, which turned out to be the electron at the energies that we are working, and established cuts that not only ensure that the process is peripheral as well as eliminate most of the background. After the cut is imposed we still have thousands of photons pairs assuming $100 \%$ efficiency in tagging the ions and detecting the photons.

The continuous $\gamma \gamma \rightarrow \gamma \gamma$ subprocess has an interesting interplay with the one resulting from the exchange of a resonance. We discuss the resonance production and decay into a photon pair. This is a nice interaction to observe because it involves only the electromagnetic couplings of the resonance. Therefore we may say that it is a clean signal of resonances made of quarks (or gluons) and its measurement is important because it complements the information obtained through the observation of purely hadronic decays. It may also unravel the possible amount of mixing in some glueball candidates [6]. We discuss the interference between these processes and compute the number of events for some specific cases.

The possibility of observing resonances that couple weakly to the photons is exemplified with the $\sigma$ meson case. This meson, whose existence has been for many years contradictory, gives a small signal in the reaction $\gamma \gamma \rightarrow \sigma \rightarrow \gamma \gamma$. However, its effects may be seen after one year of data acquisition, providing some clue about this elusive resonance. Using values of mass, total, and partial widths currently assumed in the literature, we compute the full cross section within a specific model and discuss the significance of the events. Our work shows the importance of the complete simulation of the signal and background of these processes including an analysis of possible systematic errors, indicating that two-photon final states in peripheral collisions can be observed and may provide a large amount of information about the electromagnetic coupling of hadrons.

\section{ACKNOWLEDGMENTS}

We would like to thank I. Bediaga, C. Dib, R. Rosenfeld, and A. Zimerman for many valuable discussions. One of us (C.G.R.) thanks M. de M. Leite for encouragement throughout this work and A. C. Aguilar for useful discussions. This research was supported by the Conselho Nacional de Desenvolvimento Científico e Tecnológico (CNPq) (AAN), by Fundacão de Amparo à Pesquisa do Estado de São Paulo (FAPESP) (C.G.R., J.P.V.C., A.A.N.), and by Programa de Apoio a Núcleos de Excelência (PRONEX).
[1] C.A. Bertulani and G. Baur, Phys. Rep. 163, 299 (1988); G. Baur, J. Phys. G 24, 1657 (1998); S. Klein and E. Scannapieco, hep-ph/9706358, International Conference on the Structure and the Interactions of the Photons, Egmond aan Zee, Netherlands, 1997; J. Nystrand and S. Klein, hep-ex/9711025, International Conference on Hadron Spectroscopy, Upton, NY, 1997; C.A. Bertulani, nucl-th/0011065, 241st WE-Hereaus Seminar: Symposium on the Fundamental Issues in Elementary Matter: In Honor and Memory of Michael Danos, Bad
Honnef, Germany, 2000; nucl-th/0104059, 17th Winter Workshop on Nuclear Dynamics, Park City, Utah, 2001; J. Rau, B. Müller, W. Greiner, and G. Soff, J. Phys. G 16, 211 (1990); M. Greiner, M. Vidović, J. Rau, and G. Soff, ibid. 17, L45 (1990); M. Vidović, M. Greiner, C. Best, and G. Soff, Phys. Rev. C 47, 2308 (1993); M. Greiner, M. Vidović, and G. Soff, ibid. 47, 2288 (1993).

[2] G. Baur, in Proceedings of the SBPF International Workshop on Relativistic Aspects of Nuclear Physics, Rio de Janeiro, 
1989, edited by T. Kodama et al. (World Scientific, Singapore, 1990).

[3] R.N. Cahn and J.D. Jackson, Phys. Rev. D 42, 3690 (1990).

[4] G. Baur and C.A. Bertulani, Nucl. Phys. A505, 835 (1989).

[5] A.A. Natale, Phys. Lett. B 362, 177 (1995); Mod. Phys. Lett. A 9, 2075 (1994).

[6] F.E. Close and A. Kirk, Eur. Phys. J. C 21, 543 (2001).

[7] E791 Collaboration, E. M. Aitala et al., Phys. Rev. Lett. 86, 770 (2001)

[8] J. Nystrand and S. Klein (LBNL-42524) nucl-ex/9811007, in Proceedings of the Workshop on Photon Interactions and the Photon Structure, edited by G. Jarlskog and T. Sjöstrand, Lund, Sweden, 1998.

[9] R. Engel, M.A. Braun, C. Pajares, and J. Ranft Z. Phys. C 74, 687 (1997)

[10] C.G. Roldão and A.A. Natale, Phys. Rev. C 61, 064907 (2000).

[11] R. Karplus and M. Neuman, Phys. Rev. 83, 776 (1951).

[12] B. De Tollis, Nuovo Cimento 32, 757 (1964); 35, 1182 (1965).

[13] V.B. Berestetskii, E.M. Lifshitz, and L.P. Pitaevskii, Quantum Electrodynamics (Editions Butterworth-Heinemann, Oxford, 1996), Vol. 4, p. 571.

[14] V. Costantini, B. De Tollis, and G. Pistoni, Nuovo Cimento A 2, 733 (1971).

[15] K. Mitchell, Philos. Mag. 40, 351 (1949).
[16] B. De Tollis and G. Violini, Nuovo Cimento A 41A, 12 (1966).

[17] V.N. Oraevskii, Sov. Phys. JETP 12, 730 (1961); M.Y. Han and S. Hatsukade, Nuovo Cimento 21, 119 (1961).

[18] Particle Data Group, D.E. Groom et al. Eur. Phys. J. C 15, 1 (2000).

[19] F.E. Close, G.R. Farrar, and Z. Li, Phys. Rev. D 55, 5749 (1997).

[20] ALEPH Collaboration, R. Barate et al., Phys. Lett. B 472, 189 (2000).

[21] S.F. Novaes, Phys. Rev. D 27, 2211 (1983).

[22] LAPP Collaboration, D. Alde et al., Z. Phys. C 36, 603 (1987).

[23] S. Klein and J. Nystrand, Phys. Rev. C 60, 014903 (1999).

[24] J. M. Blatt and V. F. Weisskopf, Theoretical Nuclear Physics (Wiley, New York, 1952).

[25] ARGUS Collaboration, H. Albrecht et al., Phys. Lett. B 308, 435 (1993).

[26] M. Boglione and M.R. Pennington, Eur. Phys. J. C 9, 11 (1999).

[27] A. Courau et al., Nucl. Phys. B271, 1 (1986).

[28] F. Sannino and J. Schechter, Phys. Rev. D 52, 96 (1995); M. Harada, F. Sannino, and J. Schechter, ibid. 54, 1991 (1996); Phys. Rev. Lett. 78, 1603 (1997).

[29] J.D. Jackson, Nuovo Cimento 34, 1644 (1964). 